\title{
Multiple perspectives integration for virtual reality- aided assemblability assessment in complex virtual assembly environment
}

\section{Yu Wang}

Beijing Institute of Technology

\section{Ziran Hu}

Beijing Institute of Technology

Pengyu Li

Beijing Institute of Technology

Shouwen Yao ( $\nabla$ armysw@bit.edu.cn )

Beijing Institute of Technology

Hui Liu

Beijing Institute of Technology

\section{Research Article}

Keywords: Virtual Reality, Virtual Assembly, Multi-perspective Interface, Multiple Perspectives Integration, VR-aided assemblability assessment

Posted Date: July 2nd, 2021

DOI: https://doi.org/10.21203/rs.3.rs-619301/v1

License: (c) (i) This work is licensed under a Creative Commons Attribution 4.0 International License. Read Full License 


\section{Abstract}

Virtual Reality (VR) has been proved as a promising tool for industrial design, but the traditional VR interface of first-person perspective (1PP) is not useful enough to support assemblability assessment in complex virtual assembly environments. In this paper, we proposed the multi-perspectives interface (MPI) which integrates the 1PP and the third-person perspective (3PP) using handheld World-in-Miniature (WIM). The MPI allows users to simulate the assembly operations in a natural manner similar to 1PP, while providing users with an overview of the assembly status through the WIM to assess the assemblability with superior spatial awareness. Two studies were included in this paper. The first study tested MPI in a general interaction task, which reveals stronger spatial awareness in MPI than in 1PP without the cost of losing natural interaction. The second study tested the performance, usability, and workload of MPI in an assemblability assessment task. The results show the advantages of MPI in the reachability evaluation. The contribution of this paper is exploring a novel interface for VR-aided assembly design and evaluation system.

\section{Full Text}

This preprint is available for download as a PDF.

\section{Supplementary Files}

This is a list of supplementary files associated with this preprint. Click to download.

- IJAMT.mp4 